\title{
Artigo Original \\ INSTABILIDADE ATLANTOAXIAL E HIPERFROUXIDÃO LIGAMENTAR NA SÍNDROME DE DOWN
}

\section{ATLANTOAXIAL INSTABILITY AND LIGAMENTOUS HYPERLAXITY IN DOWN SYNDROME}

\author{
Marcos Almeida Matos ${ }^{1}$
}

\section{RESUMO}

A instabilidade atlantoaxial (IAA) é caracterizada pelo aumento da mobilidade da articulação C1-C2 devido a frouxidão ligamentar alar, sendo freqüente em portadores da Síndrome de Down. O objetivo deste trabalho é estudar a freqüência de IAA e sua associação com hiperfrouxidão ligamentar generalizada em pacientes com Síndrome de Down. Selecionou-se 80 crianças, 34 (42,5\%) masculinos e $46(57,5 \%)$ femininos, com idades entre 5,6 e 15 anos (média de 9). Os pacientes foram avaliados por questionário e radiografia em perfil da coluna cervical em flexão. Encontrouse sintomatologia clínica em $58 \%$, comumente relatada como fraqueza em membros inferiores. A distância atlanto-odontoidal foi de até $4,5 \mathrm{~mm}$ em $77,5 \%$, de 4,5 a $6 \mathrm{~mm}$ em $15 \%$ e, maior que $6 \mathrm{~mm}$ em $7,5 \%$ dos pacientes avaliados. Os pacientes portadores de hiperfrouxidão ligamentar generalizada (grupo 1) não apresentaram maior freqüência de IAA em relação aos não portadores de hiperfrouxidão (grupo 2). Os autores concluíram que a IAA apresenta alta freqüência na síndrome de Down, não havendo associação direta com hiperfrouxidão ligamentar generalizada.

Descritores: Síndrome de Down; Patologia; Coluna cervical

\section{SUMMARY}

Atlantoaxial instability $(A A l)$ is characterized by hypermobility of C1-C2 joint due to laxity of alar ligament, being common in Down syndrome patients. The aim of this study is to verify $A A l$ incidence and its association with generalized ligamentous laxity in Down syndrome. Eighty children were selected, 34 (42.5\%) males and $46(57.5 \%)$ females, aged from 5.6 to 15 years-old (average $=9$ ). Patients were evaluated by questionaire and an X-ray imaging of cervical spine in a flexed lateral view. Clinical features amount to $58 \%$, most expressed as weakness of lower limbs. $77,5 \%$ of atlantoodontoid spaces were as small as $4.5 \mathrm{~mm} ; 15 \%$ were between 4.5 to $6 \mathrm{~mm}$; and $7.5 \%$ were greater than $6 \mathrm{~mm}$. Patients presenting generalized ligamentous laxity (group 1) did not show a greater incidence of AAl compared to those not presenting that condition (group 2). The authors concluded that $A A l$ is a common disorder in Down syndrome, having no direct association to generalized ligamentous laxity.

Keywords: Down syndrome; Pathology; Cervical spine.

\section{INTRODUÇÃO}

A prática esportiva em portadoras de síndrome de Down tem se consolidado como importante método de terapia, integração e sociabilização $0^{(1)}$. Entretanto, a presença de instabilidade atlantoaxial (IAA) nestas crianças merece consideração especial porque expõe os seus portadores a sérios riscos de lesão medular aguda com morte súbita, caso ocorra, durante a atividade esportiva, uma flexão cervical forçada, luxando ou subluxando as vértebras e comprimindo a medula espinhal(1)

Spitzer et al. (2) publicaram o primeiro relato de instabilidade atlanto-axial em portadores de Síndrome de Down. A partir de então, vários autores têm relatado esta associação numa taxa que varia entre $9 \%$ a $40 \%$, com apenas $10 \%$ a $15 \%$ destes indivíduos sendo sintomáticos ${ }^{(1,3,4,5,6)}$. Por este motivo, a identificação de IAA e fatores de risco nestas crianças é muito importante antes da indicação da prática esportiva. A hipoplasia do odontóide e as alterações degenerativas da coluna cervical já foram relatadas como fatores predisponentes; entretanto, a hiperfrouxidão ligamentar generalizada, que também é encontrada em altos índices nestes indivíduos, tem sido considerada por alguns autores a principal entidade relacionada com a IAA.

Sabe-se que a instabilidade atlantoaxial (IAA) é uma afecção caracterizada pelo aumento da mobilidade da articulação formada pela primeira e segunda vértebras cervicais (articulação atlantoaxial) devido a frouxidão do ligamento alar a este nível|(7). Porém a associação entre frouxidão ligamentar alar e hiperfrouxidão ligamentar generalizada, bem como seu verdadeiro significado, ainda permanecem mal definidos na literatura.

Baseado nesta controvérsia, realizou-se estudo de corte transversal que busca verificar a freqüência de IAA em portadores de síndrome de Down e sua possível associação com hiperfrouxidão ligamentar generalizada.

\section{MATERIAL E MÉTODOS}

Realizou-se estudo de corte transversal na população de indivíduos portadores da Síndrome de Down, atendidas na APAE (Associação de Pais e Amigos dos Excepcionais) da cidade de Salvador e no Serviço de Ortopedia Pediátrica da Escola Bahiana de Medicina (Hospital Santa Izabel).

O estudo teve aprovação da Comissão de Ética em Pesquisa da Escola Bahiana de Medicina. Os pais ou responsáveis pelas crianças foram contactados e informados sobre o objetivo da pesquisa e sobre quais exames seriam realizados com finalidade diagnóstica. Após permissão consentida pelos pais ou responsáveis, foram marcados os dias e horários para aplicação do questionário e avaliação radiográfica.

Foram selecionados 80 pacientes sendo 34 (42,5\%) do sexo masculino e $46(57,5 \%)$ do sexo feminino, cuja idade variou de 5,6 a 15 anos, com média de 9 anos. A distribuição dos indivíduos encontra-se sumarizada por sexo e idade na Tabela 1. 
Foi registrado todo tipo de sintomatologia importante, as condições gerais de saúde, presença ou não de desconforto ou qualquer tipo de dor e sua respectiva localização; além de detalhes sobre o desenvolvimento motor. Após a aplicação do questionário, os pacientes submetidos a exame radiográfico em perfil da coluna cervical em flexão, conforme o método de Kobori e Takahashi(8).

A avaliação das radiografias foi realizada pessoalmente pelo autor, considerando-se a mensuração da distância entre a vista pósteroinferior do arco anterior do Atlas (C1) e a superfície anterior adjacente do processo odontóide - distância atlanto-odontoidal (DAO) - segundo os critérios padronizados por Pueschel et al. ${ }^{(9)}$. Considerou-se DAO de até $4,5 \mathrm{~mm}$ como dentro dos padrões de normalidade (tipo I), DAO entre 4,5 e $6 \mathrm{~mm}$ foi considerada sugestiva de IAA (tipo II) e, finalmente, considerou-se IAA franca naqueles pacientes com valores acima de $6 \mathrm{~mm}$ (Tipo III).

Para verificação da hiperfrouxidão ligamentar generalizada utilizou-se os critérios de Wynne-Davies e Gormley ${ }^{(10)}$, que considera 5 itens relacionados com a movimentação articular: 1) Extensão do cotovelo $>180^{\circ}$; 2) Polegar alcança antebraço com punho fletido; 3) Dedos da mão ficam paralelos ao antebraço à extensão máxima de punho e metacarpofalangeanas; 4) Dorsiflexão do tornozelo $\geq 45^{\circ}$; 5) Extensão do joelho $>180^{\circ}$. Quando 3 pares quaisquer das articulações acima apresentavam estas características, considerava-se que existia hiperfrouxidão ligamentar generalizada.

Utilizou-se a estatística descritiva para apresentação dos dados e o teste do Qui-quadrado foi utilizado para avaliar significância entre dados não paramétricos nas Tabelas 1, 2 e 3, estabelecendo-se sempre _ $=0,05$ como nível de significância.

\section{RESULTADOS}

Entre os 80 portadores de síndrome de Down avaliados, encontramos $6(7,5 \%)$ crianças com DAO maior que $6 \mathrm{~mm}$ (tipo III), $12(15 \%)$ tinham DAO entre $6 \mathrm{~mm}$ e $4,5 \mathrm{~mm}$ (tipo II) e $62(77,5 \%)$ tinhamDAO menor que 4,5 (tipo l). A DAO média na população estudada foi de $4,13 \mathrm{~mm}$ e a instabilidade atlantoaxial (tipo II e III) esteve presente em $22,5 \%$ das crianças.

Havia hiperfrouxidão ligamentar generalizada em $61,2 \%$ dos indivíduos. Outros problemas ortopédicos estavam presentes em $77 \%$ dos indivíduos, sendo que $97 \%$ tinham pés planos, $50 \%$ cifose, $40 \%$ geno valgo, $20 \%$ geno varo, $20 \%$ escoliose, $15 \%$ lordose, $10 \%$ rotação interna de membros inferiores.

Quanto ao tônus, $46 \%$ das crianças tinham tônus normal e $54 \%$ apresentavam hipotonia. A diminuição da força muscular foi um sintoma encontrado em $27 \%$ das crianças. Todas as crianças praticam uma ou mais modalidades esportivas, numa freqüência maior que três vezes por semana.

Quanto à sintomatologia que poderia estar relacionada a IAA, $58 \%$ das crianças apresentavam fraqueza de membros inferiores, enquanto que apenas $12 \%$ apresentaram algum relato de perda de consciência ocasional. A limitação da movimentação cervical foi encontrada em $27 \%$, sendo

\begin{tabular}{|l|c|c|c|}
\hline \multicolumn{1}{|c|}{ Idade } & Masculino & Feminino & Geral \\
\hline Até 8 anos & 8 & 15 & 23 \\
\hline 8-12 anos & 18 & 21 & 39 \\
\hline 12-15 anos & 8 & 10 & 18 \\
\hline Total & 34 & 46 & 80 \\
\hline
\end{tabular}

$X^{2}$ calculado $=0,801, P=0,670$

Tabela 1 - Distribuição dos pacientes segundo sexo e idade.

\begin{tabular}{l|c|c|c}
\hline \multicolumn{1}{c|}{ Sexo } & DAO $<4,5 \mathrm{~mm}$ & DAO $>4,5 \mathrm{~mm}$ & Geral \\
\hline Feminino & 34 & 12 & 46 \\
\hline Masculino & 28 & 6 & 34 \\
\hline Total & 62 & 18 & 80 \\
\hline
\end{tabular}

$X^{2}$ calculado $=0,388, P=0,533$

Tabela 2 - Relação do sexo com a distância atlanto-odontoidal $(D O A)$.

\begin{tabular}{l|c|c|c}
\hline \multicolumn{1}{c|}{ DAO } & Sem hiperfrouxidão & Com hiperfrouxidão & Geral \\
\hline$<4,5 \mathrm{~mm}$ & 25 & 37 & 62 \\
\hline$>\mathbf{4 , 5 m m}$ & 6 & 12 & 18 \\
\hline Total & 31 & 49 & 80 \\
\hline
\end{tabular}

Tabela 3 - Relação entre hiperfrouxidão ligamentar generalizada e distância atlanto-odontoidal (DAO). que em nenhum destes casos havia sintomatologia álgica. Dor nos membros inferiores estava presente em $23 \%$ dos indivíduos dos casos a dor relacionava-se à coluna cerou irradiava-se para os ombros. Parestesia inespecífica foi

encontrado atraso no desenvolvimento motor em todas as . 列 $31 \%$, ou seja, com atraso em relação às crianças consideradas com desenvolvimento motor normal. O valor mais significativo encontrado foi quanto à deambulação, pois $81 \%$ das crianças ano e 4 meses.

Para efeito de análise da associação entre Hiperfrouxidão Ligamentar generalizada e IAA, os portadores de DAO tipo II e tipo III

\section{DISCUSSÃO}

Os pacientes portadores de síndrome de Down podem apresentar vários problemas ortopédicos, tais como instabilidades avenis. A maioria destes problemas encontra-se relacionada com - ligamentar generalizada que estes individuos possuem. Entretanto, os mais graves problemas ortopédicos coluna cervical $(1,3,6)$

A hipoplasia do odontóide, a artrose cervical baixa e a instabilidade C1-C2 acometem entre 10 a 20\% dos pacientes, embora apenas 1 a $2 \%$ deles sejam sintomáticos ${ }^{(1,9)}$. A presença de instabilidade atlantoaxial (IAA) merece, entretanto, consideração especial porque expõe seus portadores a sérios riscos de lesão medular aguda, especialmente durante a prática esportiva, que cada vez torna-se mais comum na conduta terapêutica e sociabilizante destas crianças.

A instabilidade atlantoaxial (IAA) também pode apresentar-se com manifestações crônicas tais como fatigabilidade fácil, dificuldades em caminhar, anormalidades da marcha, dor cervical, limitação da mobilidade cervical, torcicolo ou inclinação da cabeça, incoordenação, déficit sensitivo, espasticidade, hiperreflexia, clônus, Babinsky e outros sintomas do neurônio motor superior. Estes sinais algumas vezes se mantêm estáveis por meses ou anos; ocasionalmente progridem e ainda mais raramente podem conduzir a paraplegia, hemiplegia, quadriplegia ou morte(1).

A medida radiográfica da distância atlanto-odontoidal (DAO) tem sido considerada o indicador mais fidedigno para a avaliação da instabilidade C1-C2. Segundo Kobori e Takahashi(8), quando a distância atlanto-odontoidal (DAO), obtida através dos Raios X em perfil com flexão da cabeça, é menor que $3,5 \mathrm{~mm}$ significa que o ligamento transverso está 
intacto; quando esta distância está entre 3,5 e 5,0 mm o ligamento transverso é insuficiente; já valores acima de $5 \mathrm{~mm}$ podem indicar insuficiência dos ligamentos alares transversos ou subluxação atlantoaxial. Estes autores referem que pessoas com DAO entre 5 e $7 \mathrm{~mm}$ devem evitar atividades esportivas de alto impacto ou de flexão que pressionem a coluna cervical. Distâncias entre $7 \mathrm{e}$ $9 \mathrm{~mm}$ implicam em importante instabilidade e deve ser feito um controle radiográfico periódico para observação, órtese cervical e, principalmente, evitar-se a prática de esportes de contato. O tratamento cirúrgico só é indicado em casos de instabilidade significante, onde estejam presentes sinais e sintomas neurológicos e DAO maior que $10 \mathrm{~mm}$. Newton ${ }^{(11)}$ considerou que crianças com valores de DAO abaixo de $4,5 \mathrm{~mm}$ podem ser consideradas na faixa de normalidade, valores acima de 4,5 são indicativos de IAA

A nossa casuística constou de 80 indivíduos portadores de Síndrome de Down distribuídos por sexo e faixa etária conforme Tabela 1. Trata-se de uma das maiores amostras da literatura relativas a este tema. Não houve diferenças significativas $(p=0,05)$ em relação à idade e ao sexo dos indivíduos (Tabelas 1 e 2). No nosso estudo, a DAO maior que $4,5 \mathrm{~mm}$ foi encontrada em 22,5\% dos indivíduos, sendo que DAO maiores que 6 $\mathrm{mm}$ somente foram encontradas em 7,5\% do total. Estes dados encontram-se em concordância com a literatura estrangeira e brasileira sobre o tema.

Acredita-se que aproximadamente $15 \%$ dos indivíduos no grupo pediátrico (menores de 21 anos) que têm Síndrome de Down também são portadores de IAA, sendo a maioria destes assintomáticos ${ }^{(1)}$. A partir do primeiro relato de IAA em portadores de Síndrome de Down em 1961 por Spitzer et al.(2), vários autores têm relatado uma incidência muito semelhante, que varia entre 9 a $13 \%$, sendo que Newton ${ }^{(11)}$ encontrou o maior índice relatado, com $40 \%$ da população com Síndrome de Down apresentando DAO maior que $4,5 \mathrm{~mm}$ no seu estudo.

No Brasil, Nahas et al. ${ }^{(4)}$ relatou a incidência de IAA em 11,76\% dos 17 pacientes que estudaram, enquanto que Minatel e Campos ${ }^{(3)}$ encontram uma taxa de 15\% (6 indivíduos) de IAA em quarenta portadores de Síndrome de Down estudados. Em nenhum destes trabalhos havia portadores sintomáticos de IAA.

No nosso trabalho encontramos uma freqüência geral de $61,2 \%$ de hiperfrouxidão ligamentar generalizada na população de por- tadores de Síndrome de Down estudada. Este achado concorda com o estudo de Semine et al.(5) que relatou a existência de alta prevalência de frouxidão ligamentar em crianças com Síndrome de Down, obtendo fequência de $76,5 \%$ no seu trabalho

Burke et al. ${ }^{(12)}$ acreditam que a etiologia da IAA, na Síndrome de Down, deva-se a um defeito na estrutura do colágeno, manifestado pela hiperfrouxidão ligamentar generalizada. Burke et al. ${ }^{(12)}$, Spitzer et al. ${ }^{(2)}$ e Tishler e Martel(13) consideraram que a frouxidão ligamentar generalizada reflete-se na frouxidão do ligamento transverso atlantal. Contudo, Ohsawa et al. ${ }^{(14)} \mathrm{em}$ um estudo com 69 crianças com Síndrome de Down não encontrou esta correlação, existindo casos que, ainda quando o número de itens positivos do índice de Carter-Wilkson diminuiu de 3 para 1, a DAO manteve-se inalterada durante o período do estudo, dando a impressão que a hiperfrouxidão do ligamento transverso atlantal não pode ser avaliada isoladamente com base na hiperfrouxidão ligamentar generalizada.

Quando buscamos, em nossos dados, verificar a associação hiperfrouxidão ligamentar generalizada e IAA (Tabela 3), nota-se que não há diferença estatisticamente significante entre os grupos $(p=0,05)$, ou seja, não houve maior prevalência de hiperfrouxidão no grupo de portadores de IAA (tipo I e tipo II). Tudo leva a crer que embora a hiperfrouxidão ligamentar generalizada tenha alta prevalência nos portadores de Síndrome de Down, esta não é a causa essencial da IAA. Possivelmente o defeito geral do colágeno que propicia a insuficiência ligamentar alar, não deve ser um fator que determine isoladamente este acontecimento, sendo necessária a interferência de outros agentes etiológicos, tais como deformidades osteogênica no Atlas e no Áxis ou outros ainda não bem esclarecidos

\section{CONCLUSÃO}

A instabilidade atlântoaxial teve prevalência de $22,5 \%$ nos indivíduos portadores de Síndrome de Down e a hiperfrouxidão ligamentar teve prevalência de $61,2 \%$. A relação causal entre estas duas condições, entretanto, não foi elucidada. Acreditase que sejam necessários trabalhos posteriores que busquem evidenciar fatores genéticos, anatômicos ou biomecânicos que possam ser considerados como determinantes na gênese da IAA na Síndrome de Down.

\section{REFERÊNCIAS BIBLIOGRÁFICAS}

1. Atlantoaxial instability in Down Syndrome: subject review. American Academy of Pediatrics Committee on Sports and Fitness. Pediatrics 1995; 96:151-4.

2. Spitzer R, Rabinowitch JY, Wybar KC. Study of abnormalities of skull, teeth and leses in mongolism. Can Med Assoc J 1961: 84:567-72.

3. Minatel EM, Campos RJ. Coluna cervical na Síndrome de Down. Rev Bras Ortop 1991;26:131-6.

4. Nahas MV, Rosário AV, Nahas AB, Luza GR. Instabilidade atlantoaxial em crianças com Síndrome de Down na grande Florianópolis: um estudo piloto. Arq Catarin Med 1995: 20:149-54

5. Semine AA, Ertel NA, Goldberg MJ, Bull MJ. Cervical spine instability in children with Down Syndrome (trissomy 21). J Bone Joint Surg Am 1978; 60:649-52.

6. Taylor TKF, Walter WL. Screening of children with down syndrome for atlantoaxial (c1C2) instability: another contetious health question. Med J Austr 1996; 165:448-50

7. Wilson MD. Special considerations for the dental professional for patients with Down 's Syndrome. J Okla Dent Assoc 1994; 84:24-6.
8. Kobori M, Takahashi MY.Atlantoaxial dislocation in Down's Syndrome. Spine 1986; 11:195-200.

9. Pueschel SM, Scola FH, Perry CD, Pezzulo JC. Atlanto-axial instability in children with own Syndrome. Pediatr Radiol 1981;10:129-32.

10. Wynne-Davies R, Gormley J. Clinical and genetic patterns in osteogenesis imperfecta. Clin Orthop 1981; 159:26-35.

11. Newton RW. Atlanto-axial instability in Down Syndrome. Greater Manchester Down's Syndrome Association - www. downsnet.org, acesso em junho de 2004

12. Burke SW, French HG, Roberts JM, Jonhston CE, Whitecloud TS, Edmund JO Chronic Atlanto-axial instability in Down Syndrome. J Bone Joint Surg Am 1985; 67:1356-60.

13. Tishler JM, Martel W. Dislocation of atlas in mongoloism. Radiology 1965;84:9048.

14. Ohsawa T, Izawa T, Kuroki Y, Ohnari K. Follow-up study of Atlanto-axial Instability in Down's Syndrome without Separate Odontoid Process. Spine 1989; 14:1149-53. 